\title{
Balantidium coli-induced pulmonary haemorrhage with iron deficiency
}

\author{
A Koopowitz, P Smith, N van Rensburg, A Rudman
}

Background. Balantidium coli, a ciliated protozoan parasite that infects primates and pigs, and is the largest protozoan to infect humans, is a well-known cause of diarrhoea and dysentery in humans. Extra-intestinal disease is uncommon, however.

Objective. We describe a case of lung involvement, with severe pulmonary haemorrhage resulting in iron deficiency anaemia and respiratory failure, of a 20 -year-old, immune-competent man.

Results. Diagnosis was made by bronchial biopsy and lavage, which showed numerous trophozoites compatible with $B$. coli with a background of acute inflammatory cells. The origin of infection was not clear, but inhalation of pig manure was postulated as there was no history of intestinal disease. The patient was treated with oxytetracyline and metronidazole, kept in an ICU, improved within 48 hours, and was discharged within 4 days.

Conclusion. B. coli infection should be considered as part of the differential diagnosis of pulmonary haemorrhage.

S Afr Med J 2010; 100: 534-536.
A case of lung involvement with Balantidium coli complicated by severe pulmonary haemorrhage resulting in iron deficiency anaemia has not, to our knowledge, been described previously.

A previously well 20-year-old white man presented to his general practitioner complaining of a gradual deterioration in his effort tolerance over the preceding month. He was pale, and a blood count showed haemoglobin $(\mathrm{Hb})$ of $9.2 \mathrm{~g} / \mathrm{dl}$, a mean corpuscular volume (MCV) of $77.8 \mathrm{fl}$ and platelet count of 345 $x 10^{9} / 1$; the other red and white cell indices were normal. Iron studies showed a reduced serum iron level of $3.8 \mu \mathrm{mol} / 1$ (normal 11.6 - $31.3 \mu \mathrm{mol} / \mathrm{l}$ ), transferrin $3.5 \mathrm{~g} / 1$ (normal 2.2 - $3.7 \mathrm{~g} / \mathrm{l}$ ), iron saturation $4 \%$ (normal $20-50 \%$ ) and ferritin $57 \mathrm{ng} / \mathrm{ml}$ (normal $20-250 \mathrm{ng} / \mathrm{ml}$ ). Serum folate and vitamin $B_{12}$ levels were normal. Iron replacement therapy was commenced per os, and the patient was referred to a surgeon with a diagnosis of iron deficiency anaemia secondary to putative gastrointestinal bleeding.

The patient was admitted to hospital 3 days later after mild haemoptysis. The $\mathrm{Hb}$ had decreased to $6.6 \mathrm{~g} / \mathrm{dl}$ and MCV to $74 \mathrm{fl}$. The international normalised ratio (INR) was 1.4. Urea and electrolytes, creatinine, liver functions, thyroid stimulating hormone, brain natriuretic peptide (pro-BNP) and alpha-1antitrypsin were normal. A semi-quantitative procalcitonin determination showed a mildly elevated level of $0.5-1.99 \mathrm{ng} / \mathrm{ml}$ (BRAHMS PCT-Q rapid assay). HIV and rheumatoid factor were negative. He gave no history of diarrhoea, abdominal

Physician in private practice, Southernwood, East London A Koopowitz, MB BCh, FCP (SA)

Ampath Laboratories, Universitas, Bloemfontein

P Smith, MB ChB, DOH, DTM\&H, MMed (Micro)

N van Rensburg, MB ChB, MMed (Clin Path), FCPath (Micro)

Ampath Laboratories, Southernwood, East London

A Rudman, BM BCh, MMed (Anat Path)

Corresponding author: P Smith (smithp@ampath.co.za) pain or haemoptysis before admission. Dark green/black stools for the preceding few days appeared to be related to his iron replacement therapy. He was hypoxic, but the oxygen saturation improved from $45 \%$ to $92 \%$ on $40 \%$ oxygen administered via a face mask. A sinus tachycardia of $120 / \mathrm{min}$ and a loud S3 gallop were present. The jugular vein pressure was not elevated. Chest X-ray showed extensive bilateral alveolar infiltrates (Fig. 1). Respiratory crackles were present bilaterally on auscultation. Gastroscopy was normal, and tests for faecal occult blood were negative.

His respiratory status deteriorated and he was transferred to the intensive care unit, but was not intubated. A low arterial $\mathrm{PO}_{2}$ with normal $\mathrm{PCO}_{2}$, compatible with Type 1 respiratory failure, improved on Bi-pap with $40 \%$ oxygen. The echocardiograph revealed normal left ventricular function with no significant valvular disease.

Blood and sputum were collected and empiric broadspectrum antibiotic and intravenous steroid therapy was initiated. Despite a blood transfusion, his respiratory status and chest X-rays showed deterioration. Sputum samples were blood-stained, but negative for acid-fast bacilli and Pneumocystis jiroveci, and only mixed oral flora were cultured. Mycoplasma pneumoniae serology testing was negative. Although the anti-nuclear factor was positive (titre 1/160), the rheumatoid factor and extractable nuclear antibody screening tests were negative, while basement membrane antibodies and neutrophil cytoplasmic antibodies were not detected. CT scanning revealed extensive bilateral para-hilar ground glass opacification in keeping with diffuse pulmonary haemorrhage (Fig. 2). A bone marrow biopsy and aspirate confirmed an iron deficiency anaemia.

Bronchial biopsy showed severe pulmonary haemorrhage and haemosiderosis and ciliated respiratory epithelium with foci of squamous metaplasia and a mixed inflammatory cell infiltrate including neutrophils. No eosinophils were noted. There was cuboidal metaplasia of the alveolar epithelium, evidence of recent haemorrhage in the surrounding lung parenchyma (Fig. 3), and alveoli with numerous haemosiderin- 


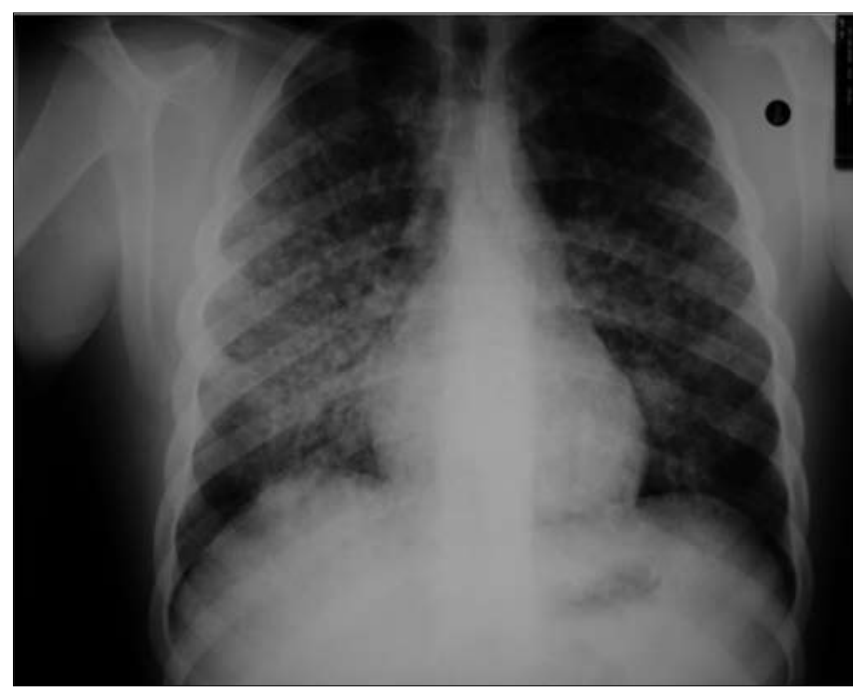

Fig 1. Chest X-ray on admission.

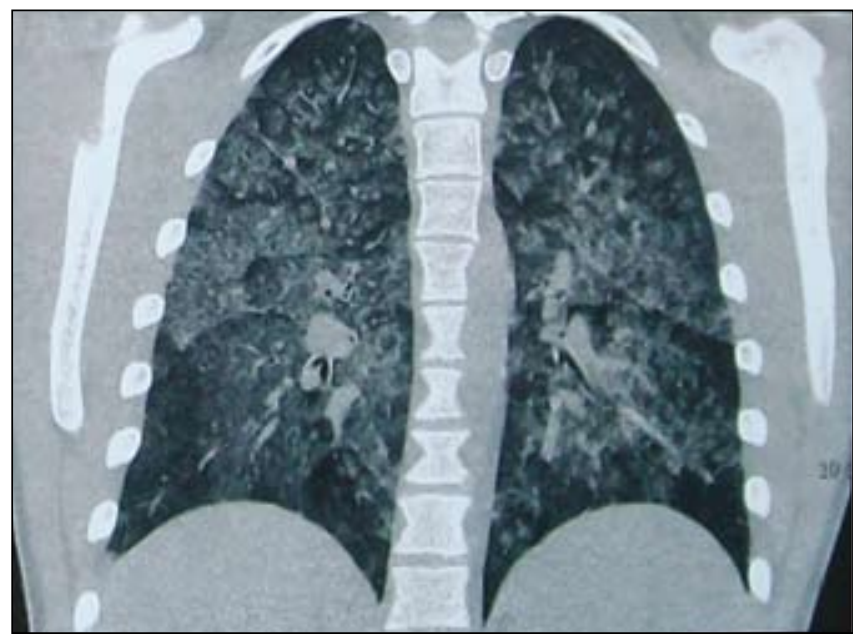

Fig. 2. CT scan of the chest.

containing histiocytes, and the Prussian blue stain for iron confirmed severe pulmonary haemosiderosis (Fig. 4). Occasional trophozoites compatible with B. coli were present.

Microscopic examination of wet preparations of the bronchial lavage fluid showed numerous ciliated $B$. coli trophozoites with a background of acute inflammatory cells. Trophozoites with typical features of B. coli were also seen in the Papanicolaou stain (Fig. 5).

Treatment with oxytetracyline $500 \mathrm{mg}$ 6-hourly and metronidazole $400 \mathrm{mg}$ 8-hourly was commenced. Within 48 hours, the patient had improved substantially and respiratory support could be stopped, and within 4 days he was well enough to be discharged. A chest X-ray after 2 months was normal (Fig. 6).

\section{Discussion}

B. coli, a ciliated protozoan parasite, infects primates and pigs, and is the largest protozoan to infect humans. Pigs may be the primary reservoir; consequently, balantidiasis is a greater risk among pig or pig manure handlers. ${ }^{1,8}$

The asexual life-cycle consists of cysts or trophozoites. Transmission is direct with no intermediate host involvement.

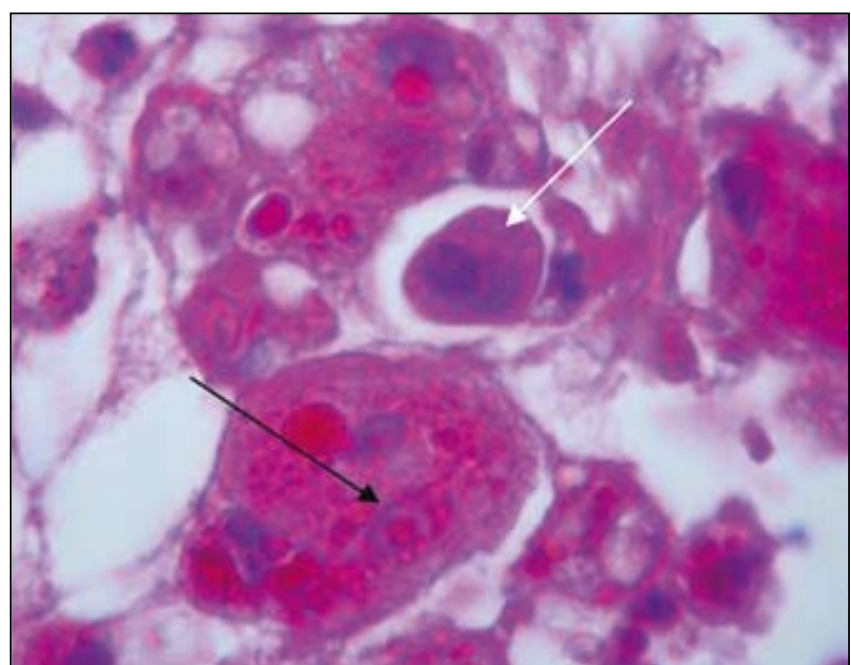

Fig. 3. Haemosiderin-containing histiocytes in alveolus (black arrow) with trophozoites (white arrow).

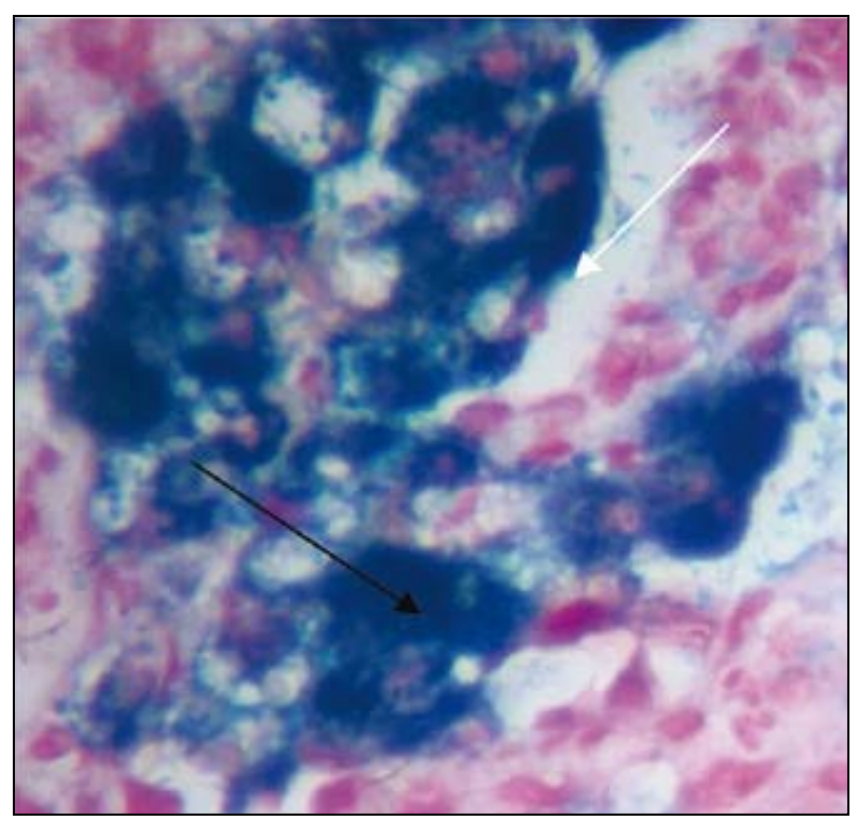

Fig. 4. Prussian blue stains for iron, indicating severe pulmonary haemosiderosis.

Usually, humans ingest infective cysts in contaminated food or water that develop into trophozoites and migrate to the large intestine, which is the organ mostly affected. The organism produces no known toxins, but can penetrate the mucosa and cause ulcers, probably owing to the production of hyaluronidase. ${ }^{2}$ Tetracyclines and metronidazole are the treatments of choice. ${ }^{1,3}$

Human infection is usually associated with intestinal symptoms such as diarrhoea and dysentery, but ranges from an asymptomatic carrier state through a chronic symptomatic infection presenting with non-bloody diarrhoea, to a dysenterylike picture.

Extra-intestinal spread to the peritoneal cavity, genitourinary tract or lungs is rare. Few cases of lung involvement with B. coli have been reported..$^{4-7}$ Respiratory tract infection in patients with an association with pigs or pig manure has been reported, probably through aerolisation of the manure., ${ }^{4,5}$ Immunocompromised individuals appear to be less resistant to 


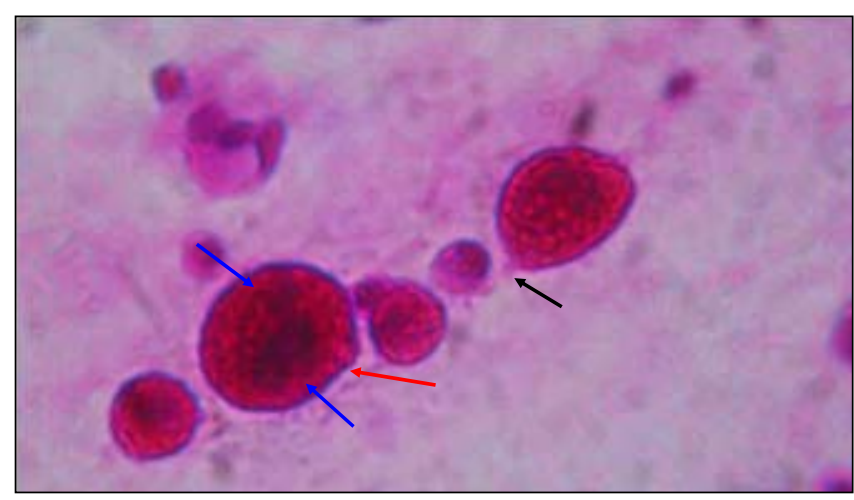

Fig. 5. B. coli trophozoites in bronchial lavage (Papanicolaou stain). Note the bean-shaped macronucleus, smaller micronucleus (blue arrows) and the region of the gullet (black arrow). Cilia are indicated by the red arrow.

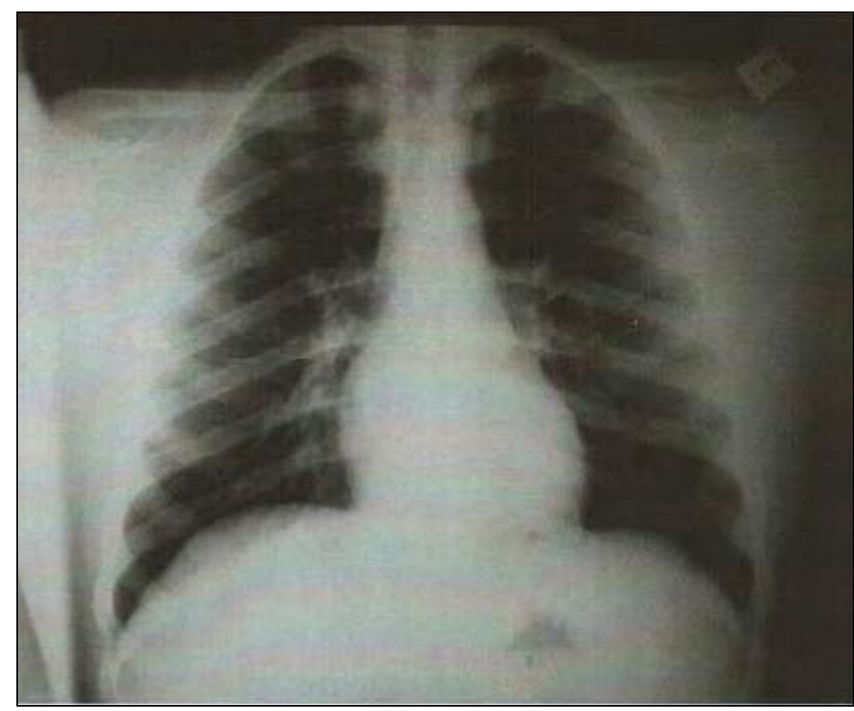

Fig. 6. Chest X-ray at follow-up after 2 months.

balantidiasis. ${ }^{2}$ Pulmonary involvement has been reported in a leukaemic patient and a patient with anal cancer. ${ }^{5,6}$

Lung involvement with $B$. coli varies. Necrotising lung infection and lung cavities have been reported in 2 cases..$^{2,7}$ In
2 immunocompromised patients, one had pleural effusions, perihilar infiltrates and diffuse consolidation in the lungs, and the other had diffuse micronodular and interstitial lung lesions. ${ }^{5,6}$ Lung tissue microscopy from a fatal case revealed $B$. coli and peri-arterial inflammatory infiltrations. ${ }^{3}$ Although $B$. coli could not be demonstrated in the lungs of a fatal case of intestinal balantidiasis, the haemorrhagic areas present were so unusual as to suggest direct parasitic involvement. ${ }^{9} \mathrm{We}$ were unable to find cases associated with diffuse pulmonary haemorrhage associated with the organism in the lungs, as in our patient.

The origin of infection in this patient is not clear. Spread most probably occurs from the intestines. ${ }^{6,7}$ Our patient had no history of intestinal disease, but unfortunately his stools were not investigated for the presence of the organism. For infection, history of an association with pigs is not always necessary. ${ }^{6,7} \mathrm{~B}$. coli has been found among the normal mouth flora, but the role in infection is unclear. ${ }^{8}$ Although the patient denied any contact with pigs or pig manure and had no significant farm exposure, he played rugby. The possibility exists that infection followed inhalation or ingestion of pig manure used to fertilise the grass.

References

1. Schuster FL, Ramirez-Avila LR. Current world status of Balantidium coli. Clin Microbiol Rev 2008; 21: 626-638.

2. Sharma S, Harding G. Necrotizing lung infection caused by the protozoan Balantidium coli. Can J Infect Dis 2003; 14(3): 163-166.

3. Mandell GL, Bennett JE, Dolin R. Mandell, Douglas and Bennett's Principles and Practice of Infectious Diseases. 6th ed. Philadelphia: Elsevier, 2005: 3232-3233.

4. Dorfman S, Rangel O, Bravo LG. Balantidiasis: report of a fatal case with appendicular and pulmonary involvement. Trans R S Trop Med Hyg 1984; 78: 833-834.

5. Vasilakopoulou A, Dimarongona K, Samakovli A, Papadimitris K, Avlami A. Balantidium coli pneumonia in an immunocompromised patient. Scand J Infect Dis 2003; 35(2): 144-146.

6. Anargyrou K, Petrikkos GL, Suller MTE, et al. Pulmonary Balantidium coli infection in a leukemic patient. Am J Hematol 2003; 73: 180-183.

7. Ladas SD, Sotiria S, Frydas A, Kaloviduris A, Hatzioannou J, Raptis S. Invasive balantidiasis presented as chronic colitis and lung involvement. Dig Dis Sci 1989; 34(10): 1621-1623.

8. Rivera F, Medina F, Ramirez P, Alcocer J, Vilaclara G, Robles E. Pathogenic and free-living protozoa cultured from the naso-pharyngeal and oral regions of dental patients. Environ Res 1984; 33: 428-440.

9. Bel GS, Couret M. Balantidium coli infection in man. J Infect Dis 1910; 7(5): 609-624.

Accepted 24 November 2009. 